\title{
DOMÓTICA COMO AUXÍLIO PARA PESSOAS COM DEFICIÊNCIA E IDOSOS
}

\section{ARTIGO ORIGINAL}

DANTAS FILHO, Carlos Alberto ${ }^{1}$, BRAZ, Daniel da Silva², ZWIPP, Gabriel Nunes ${ }^{3}$, SANTOS, Lukas Gabriel Assis $\operatorname{dos}^{4}$, MELO, Thiago Cunha de ${ }^{5}$, BENITES, Cristiano da Silva ${ }^{6}$

DANTAS FILHO, Carlos Alberto. Et al. Domótica como auxílio para pessoas com deficiência e idosos. Revista Científica Multidisciplinar Núcleo do Conhecimento. Ano 06, Ed. 04, Vol. 02, pp. 118-131. Abril de 2021. ISSN: 2448-0959, Link de acesso: https://www.nucleodoconhecimento.com.br/educacao/domotica, DOI: 10.32749/nucleodoconhecimento.com.br/educacao/domotica

\section{RESUMO}

A automação é um ramo de estudo que teve início no setor industrial, depois em edifícios comerciais e agora na domótica. Esta tecnologia aplica-se ao ambiente doméstico para ser controlada pelo usuário por meio de um aplicativo ou comando de voz, dando vida aos seus serviços, conforto e comodidade aos seus usuários, tudo isso podendo ser realizado via celular. A automação residencial é um conjunto completo de serviços técnicos, visando a melhor maneira de atender às necessidades básicas de segurança, comunicação, gestão de energia e conforto de vida. A casa inteligente é como uma casa com vida própria, por isso o sistema de automação residencial tem as características de um sistema inteligente, que pode interagir com os moradores. Adotamos métodos de pesquisas bibliográficas

\footnotetext{
${ }^{1}$ Graduação em ciência da computação em andamento.

${ }^{2}$ Graduação em ciência da computação em andamento.

${ }^{3}$ Graduação em ciência da computação em andamento.

${ }^{4}$ Graduação em ciência da computação em andamento.

${ }^{5}$ Graduação em ciência da computação em andamento.

${ }^{6}$ Orientador. Mestrado em Engenharia Elétrica e Computação. Pós-Graduação MBA em Data Center e Computação em Nuvem. Graduação em Ciência da Computação e Tecnólogo em Redes de Computadores.
}

RC: 81825

Disponível em: https://www.nucleodoconhecimento.com.br/educacao/domotica 
baseados em materiais já publicados, composto por livros e artigos científicos, bem como pesquisas exploratórias para proporcionar a maior familiaridade com a automação residencial (AR), especificamente para pessoas com deficiência (PCD). Com este artigo, pretendemos apresentar os principais aspectos da residência automática, enfocando elementos básicos e típicos. Diferentes campos e aplicações também são considerados, enfatizando os diversos benefícios que a casa automática pode trazer, como: acessibilidade, comodidade, praticidade, sistemas de segurança e controle de gastos, controle de luzes, portas automatizadas, janelas, camas e escadas. Apontamos, também, algumas desvantagens como: alto custo e má utilização, sempre com foco em PCD. A pesquisa destaca diversos aspectos relevantes do planejamento e aplicações de automação residencial para apoiar os vários elementos de casas automatizadas.

Palavras-chave: Automação residencial, idoso, automático, qualidade, deficiência.

\section{INTRODUÇÃO}

Historicamente de acordo com Adami (2011), podemos dizer que a automatização começou primeiramente com a automação industrial e depois de prédios comerciais, só então veio a automatização de residências sendo ela o que aplica tecnologia no ambiente doméstico com a finalidade de trazer mais conforto e facilidade nos serviços dos mesmos sendo controlados através de controles remotos ou até mesmo de via internet através de aparelhos celulares.

Automatização Residencial é o conjunto de serviços tecnológicos integrados como o melhor meio de satisfazer as necessidades básicas de segurança, comunicação, gestão energética e conforto de uma residência, conforme Aureside (2013). Esse tipo de tecnologia ainda é algo novo e o preço ainda está em processo de se tornar mais acessível para as pessoas.

Conforme a tecnologia foi evoluindo ela foi indo para várias outras áreas, como economia, plantios, mercados, indústria e muitos outros, mas o que realmente 
vamos focar é nas áreas da saúde, pois é uma área que a tecnologia vem crescendo e muito, ela ajuda no estudo dos futuros médicos e também consegue fazer microcirurgias, o que antes precisava abrir a pessoa , hoje em dia com uma máquina você consegue fazer um furo mínimo e tratá-la sem precisar abrir tanto. (ELOY et al., 2015)

Conforme Dino (2014), com o aumento da expectativa de vida da população, o aumento de idosos, o desejo de uma independência pessoal e com os problemas que elas tem no seu dia a dia, por motivos de idade ou alguma deficiência que elas tem, são fatores que contribuem para a automação residencial dessas pessoas, pois elas terão sua independência pessoal, além de mais conforto e facilidade em fazer certas ações.

Sendo assim, esse trabalho tem como objetivo mostrar como a domótica pode ser um grande auxílio paras as pessoas com idade avançada ou com alguma deficiência, mostrando como elas podem ter sua independência e maior conforto implementando-a em sua vida.

\section{DOMÓTICA}

\subsection{O QUE É DOMÓTICA}

A domótica pode ser definida como um conceito de integração dos mecanismos automáticos em um determinado espaço. É uma tecnologia recente e é responsável pela gestão de todos os recursos habitacionais. Encontrando soluções para as nossas necessidades do modo mais prático e com menos esforço possível. (LINS et al., 2009)

O termo domótica surgiu na França por volta do século 20, e tem uma fusão da palavra "Domus", que significa "casa", com a palavra "Robótica", historicamente pode-se dizer que a automatização começou com a automatização industrial e depois automatização de prédios comerciais. É uma tecnologia que antigamente

RC: 81825

Disponível em: https://www.nucleodoconhecimento.com.br/educacao/domotica 
poucos tinham acesso, mas com a evolução da tecnologia ficou mais fácil tela dentro de casa. (SANTOS et al., 2016)

A domótica, de acordo com se aplica em várias partes de uma casa como iluminação, climatização, som ambiente, segurança, comunicação e conforto. Ela pode melhorar e facilitar a qualidade de vida de muitas pessoas com dispositivos controlados por voz ou com a análise de nossas ações. (SGARBI et al., 2011)

\subsection{DOMÓTICA EM GERAL}

Para Beleza (2009), domótica é o controle automatizado de casas, definida como integração entre os mecanismos automáticos em um espaço, auxiliando e deixando mais prático o cotidiano das pessoas, sendo aplicada no conforto, segurança e entre outros, é uma tecnologia recente e é responsável pelo controle de todos os recursos habitacionais.

De acordo com Beleza (2009), de forma mais abrangente, automação residencial poder ser entendida como a reunião de conhecimentos, alterando a observação, esforço e processo de decisão humano por dispositivos eletrônicos, mecânicos e softwares gerados por meio da tecnologia.

A definição de domótica, é a utilização simultânea da eletricidade e das tecnologias da informação no ambiente residencial, permitindo realizar a sua gestão, local ou remota, e oferecer uma vasta gama de aplicações nas áreas de segurança, conforto, comunicação e gestão de energia. (MARIOTONI et al., 2007)

Segundo Nunes (2002), pode-se dizer que as características fundamentais de um sistema inteligente é ter memória, ter noção temporal, fácil interação com que a utiliza, interação entre todos os sistemas do ambiente, facilidade de reprogramação e capacidade de autocorreção.

A Domótica inteligente não é somente uma residência com um sistema dotado de controle central que possa melhorar e facilitar certas funções. A residência

RC: 81825

Disponível em: https://www.nucleodoconhecimento.com.br/educacao/domotica 
inteligente é algo como uma residência com vida própria, portanto os sistemas de Domótica inteligente apresenta as características de um sistema inteligente interagindo com os habitantes da residência, fazendo com que aprenda dinamicamente com seus comportamentos. (SGARBI et al., 2011)

\subsection{VANTAGENS E DESVANTAGENS}

Segundo Teixeira (2015), na domótica é possível notar inúmeras vantagens e poucas desvantagens, pois a tecnologia vem nos trazendo sempre inovação nas atividades do nosso dia a dia, desde as mais simples como até as mais complexas, tais quais os próprios seres humanos quem os faz, não tem a capacidade de fazer a atividade do mesmo. Por isso que a tecnologia está em alta e existe uma constante pesquisa para se ter novas descobertas ou até mesmo aprimorar e inovar, tudo em prol do conforto, praticidade, agilidade, velocidade e a possibilidade de tornar possível a realização de tarefas que antes difíceis ou impossíveis de fazê-lo, pessoas com uma idade mais avançada ou até mesmo com a mobilidade reduzida seria capaz de fazer.

Domótica é uma tecnologia excepcional e que já é realidade, mas pouco utilizada ao se analisar a quantidade de vantagens e benefícios que ela traz. Com o bom uso da domótica é possível ter mais segurança pois poderia ter travas de seguranças automáticas ou pré-programadas, câmeras de segurança com sensores e detectores faciais e de movimento, também com possível acesso das imagens pelo celular ou uma conta na internet de qualquer lugar que se esteja, podendo assim acompanhar a casa de longe e sendo informado a qualquer movimento indevido ou que estejam fora do padrão, pelo mesmo sistema seria possível também controlar os gastos de energia, trazendo assim vantagens ao meio ambiente e sustentabilidade, sem falar nas atividades em geral que poderiam ser de fácil controle onde até mesmo pessoas que tenham deficiência física ou mobilidade reduzida e idosos poderiam fazer suas atividades dentro de casa sem depender de uma outra pessoa, tornando assim de certo modo independente de pessoas presentes o tempo todo ou para tudo, mas

RC: 81825

Disponível em: https://www.nucleodoconhecimento.com.br/educacao/domotica 
com o auxílio da tecnologia em seu lugar, assim sensores e pré-programarão ou até mesmo sistemas por comando de voz iria tornar muitas coisas possíveis, trazendo mais qualidades de vida para essas pessoas. (BERNINI et al., 2016)

Conforme Domingues (2013), tratando-se dos pontos negativos da domótica, não é a tecnologia em si que se tenha problemas, mas sim de como ela pode ser mal utilizada ou utilizada de forma exagerada e que não é uma tecnologia sempre presente como deveria ou até mesmo não de fácil acesso as pessoas de classe mais baixa, isso quer dizer que pelo fato de ser algo moderno e uma novidade e em constante aprimoramento, o custo do mesmo acaba se tornando de não tão fácil de ter-se posse dessa tecnologia. Tratando se qualidade de vida o mal-uso dessa tecnologia pode torná-las pessoas saudáveis mal-acostumadas e muito dependentes dessas facilidades e se acomodando de mais a ponto de não fazer quase nenhum esforço físico, podendo entanto trazer problemas a saúde.

Se formos analisar os dois lados, sendo eles as vantagens e as desvantagens podemos de longe nota que a quantidade de coisas positivas se destaca em relação ao que se tem de negatividade, e vale realmente a pena investir mais e continuar investindo nisso com o objetivo dessa tecnologia alcançar e se tornar possível para o uso de toadas as pessoas, sendo para auxilio básico ou para algo maior que vai além e melhore o conforto e segurança da pessoas.

\subsection{APLICAÇÃO DA DOMÓTICA PARA PESSOAS IDOSAS E DEFICIENTES}

A automação residencial tem tornado a vida das pessoas muito mais prática e confortável para realizar tarefas comuns do dia a dia. Já é possível automatizar qualquer recurso eletrônico, por exemplo, o simples ato de abrir a porta já pode ser feito através do celular. Não vivemos mais no tempo em que a automação residencial era vista como item de luxo.

$\mathrm{RC}: 81825$

Disponível em: https://www.nucleodoconhecimento.com.br/educacao/domotica 
Hoje, de acordo com Martins (2019), esses recursos podem se tornar imprescindíveis à acessibilidade e independência de pessoas com deficiência, permitindo que elas possam realizar tarefas cotidianas, que antes eram consideradas complexas e até mesmo impossíveis. Uma casa adaptada significa também tornar a vida de portadores de deficiência física, ou pessoas com dificuldade de locomoção, como idosos e obesos mais fácil e pode-se dizer até que mais felizes e prazerosas.

A popularização da tecnologia já a torna também muito mais acessível ao consumidor comum. De acordo uma pesquisa oficial, o Sistema Único de Saúde nos trouxe os seguintes dados, $75 \%$ das quedas de pessoas de mais idade acontecem dentro de casa. Além disso, de acordo com pesquisas, no Brasil cerca de $30 \%$ dos idosos caem ao menos uma vez por ano e de $5 \%$ a $10 \%$ destes sofrem lesões severas. Uma casa automatizada significa mais conforto, segurança e funcionalidade para pessoas nessas condições, já que não é necessário se levantar ou locomover para controlar iluminação, climatização, sistema de entretenimento, acesso, e muitas outras tarefas. (PERRACINI, 2002)

O gráfico abaixo mostra em detalhes a taxa de crescimento da população idosa no Brasil e no mundo, desde 1950 até, como previsão, 2020. (PAIVA et al., 2008)

RC: 81825

Disponível em: https://www.nucleodoconhecimento.com.br/educacao/domotica 
Figura 1 - Crescimento da população idosa

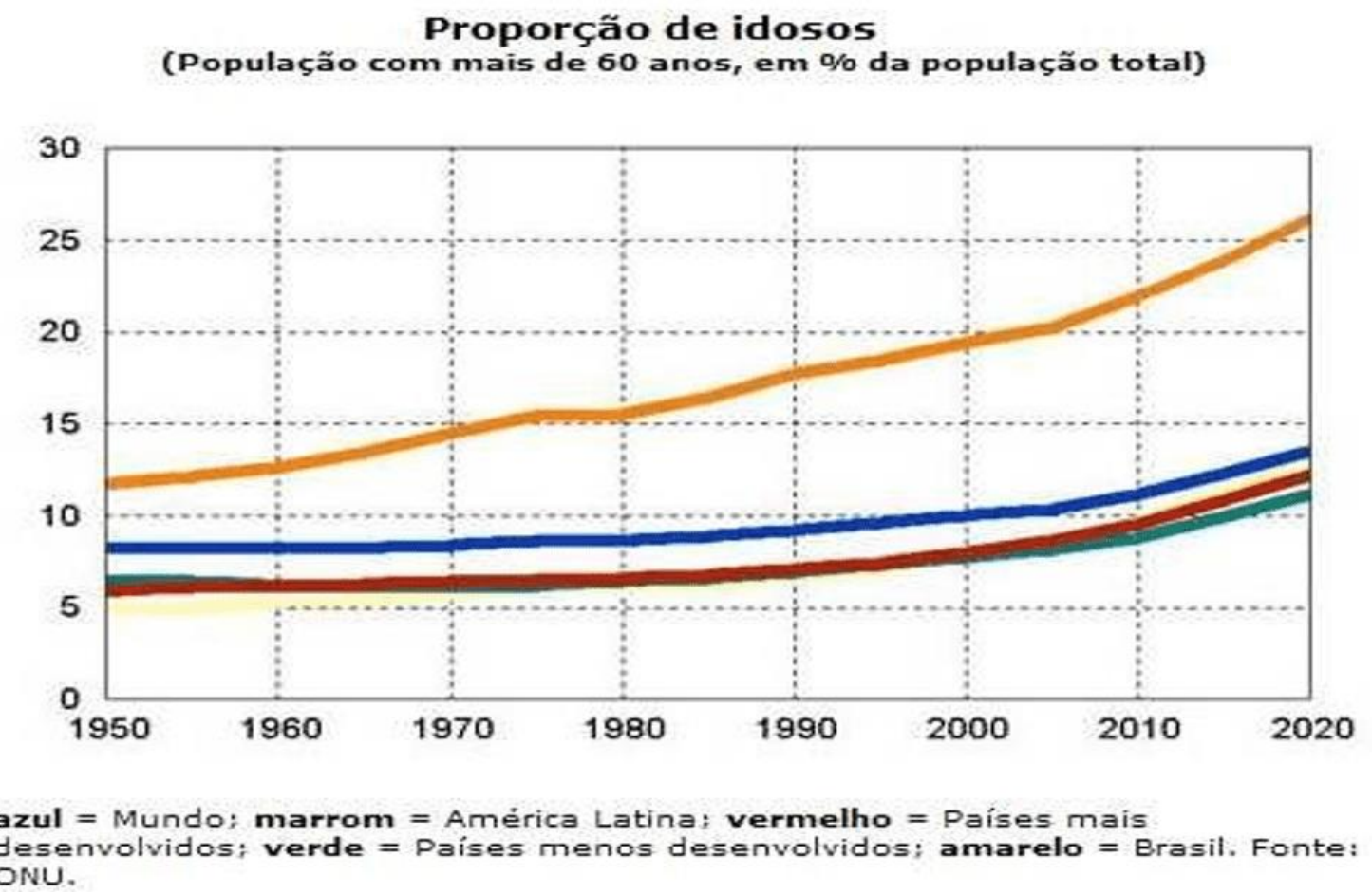

Fonte: http://america8bmanha.blogspot.com/2009/05/expectativa-de-vida-naamerica-latina.html

Limitações físicas funcionais em pessoas com deficiência e de idade mais avançada podem ser manifestadas de infindas formas, e em sua grande maioria, essas formas de deficiência podem colocar de lado indivíduos da sociedade, incluindo do mercado de trabalho. O Ministério do Trabalho e Emprego divulgou uma estatística em 2013 mostrando que dos 49 milhões de vínculos trabalhistas ativos, apenas 358 mil eram de pessoas deficientes $(0,73 \%$ no total), sendo que destas $51 \%$ representavam pessoas com deficiências físicas. Devido ao alto custo e complexidade no desenvolvimento de tecnologias que promovem a inclusão social, o deficiente tem grandes dificuldades em suas atividades diárias e permanece excluído da sociedade e do trabalho. A utilização da automação permite a padronização e a flexibilização de sistemas, fazendo com que seja possível utilizá-la dentro do ambiente doméstico 
como ponto positivo para a melhora na qualidade de vida das pessoas. (BERNINI et al., 2016)

As plataformas Arduino (plataforma de programação através de microcontroladores), muito utilizado em simples e grandes projetos, o que torna possível sua interação com o celular através da plataforma Android, que pelo fato de ser código aberto, gratuito e não estar preso a um hardware específico possui uma quantidade imensa de aplicações e aplicativos disponíveis, podendo eles serem programados em blocos ou em linguagens de programação como Java. o que nos traz duas plataformas perfeitas para criar sistemas e novas tecnologias em prol da domótica em casas adaptadas para pessoas cujo necessitam de cuidados especiais. (JÚNIOR et al., 2015)

Acrescentando o fato de que existe mercado consumidor crescente, visando o crescimento da expectativa de vida.

Podemos apresentar algumas aplicações e tecnologias a serem implementadas, como por exemplo:

\subsubsection{TELEMEDICINA}

Para Nichele (2016), a medicina aliada à telemetria pode monitorar pessoas em suas próprias residências, assim não apenas evitando que tenham que se deslocar até um posto médico, como também sendo um apoio à medicina preventiva. Possibilitaria o monitoramento de cardíacos 24 horas por dia, sendo os dados transmitidos em tempo real para o hospital através da mesma rede de acesso que viabiliza a conexão com a Internet, assim como, por exemplo, permitiria aos diabéticos o envio dos resultados dos seus exames diários por meio de um leitor de glicose integrado ao sistema residencial automatizado. Ao passo que, no hospital, esses resultados seriam armazenados num banco de dados, sendo o médico avisado pelo software gerenciador sobre qualquer disfunção apresentada nos dados.

$\mathrm{RC}: 81825$

Disponível em: https://www.nucleodoconhecimento.com.br/educacao/domotica 


\subsubsection{CAMA DE BANHO}

Consiste num equipamento portátil de higienização para pessoas acamadas que é composto por uma bacia e um gabinete de serviço e controle. Assim, tal estrutura é especialmente projetada para proporcionar à pessoa conforto e segurança. Nesta, a bacia é posta sob a pessoa em sua própria cama, enquanto o gabinete de serviço e controle possui um sistema de acondicionamento, aquecimento e bombeamento de água para o banho, assim como um mecanismo de deslize sobre rodas que, durante o banho, fica ao lado da cama. Trata-se de um sistema especial de absorção e escoamento da água usada, pelo qual o assistente consegue dar um banho completo no paciente, sem desperdícios e com grande economia de água. (NICHELE, 2016)

\subsubsection{CAMA ARTICULÁVEL}

Cama com controle remoto onde o usuário poderá articular a cama para que se sinta mais confortável. (NICHELE, 2016)

\subsubsection{FRASCOS PARA ARMAZENAMENTO DE REMÉDIOS}

De acordo com Nichele (2016), esse sistema compõe-se pela tampa do frasco e por uma estação com aparência de uma lâmpada. Quando esse frasco é aberto pelo usuário um sinal wireless (sem fio) é emitido para uma base que envia mensagens para um computador ou para um celular pré-estabelecido através de uma rede. Caso o frasco não seja aberto no horário estipulado, a tampa possui um mecanismo de luz que começa a piscar, a fim de lembrar ao paciente que ele esqueceu o remédio. Na eventualidade desse mecanismo não funcionar, o produto também emite sons. Assim, por fim, o sistema ainda pode enviar uma mensagem como um tipo de lembrete, bem como ele também pode encaminhar esse alerta para outras pessoas, como parentes e médicos, de modo a "dedurar" o paciente.

RC: 81825

Disponível em: https://www.nucleodoconhecimento.com.br/educacao/domotica 


\subsubsection{PERSIANA COM CONTROLE REMOTO}

Segundo Ferreira (2010), o uso remoto de persianas é um sistema de fácil instalação e utilização que permite ao usuário maior comodidade. Assim, a instalação desse sistema reduz a necessidade da locomoção do usuário até a persiana para fechá-la, percurso esse muitas das vezes traçado por móveis e objetos. O Acionamento Remoto de Persianas é de grande utilidade para pessoas enfermas.

\subsubsection{PLATAFORMAS PARA CADEIRAS}

De acordo com Rangel (2013), esse tipo de plataforma permite o usuário impossibilitado de subir escadas, transpassá-las sem nenhum problema. A plataforma é usada principalmente em pequenas escadas, em entradas de casas ou na parte interna das residências. Edifícios como as Agências Bancárias, onde possuem pequenas escadas, são obrigadas por lei, disponibilizar esse tipo de equipamento. Esse tipo de a equipamento suporta até $250 \mathrm{Kg}$.

\subsubsection{PLATAFORMAS PARA IDOSOS E NÃO CADEIRANTES}

Conforme Domingues (2013), essas plataformas são utilizadas sobretudo por pessoas que possuem dificuldades para subir escadas. Trata-se de um assento com motor e controle manual que permite ao usuário o controle da cadeira em subir, descer ou parar a mesma quando desejar. Esse tipo de equipamento suporta até $150 \mathrm{Kg}$.

\subsubsection{ELEVADORES VERTICAIS}

Segundo Domingues (2013), os elevadores residenciais são equipamentos que também poderão ser instalados no interior ou no exterior das casas. Por ser um equipamento de alto custo, ele ainda não é muito utilizado. Consiste num elevador

$\mathrm{RC}: 81825$

Disponível em: https://www.nucleodoconhecimento.com.br/educacao/domotica 
fechado que poderá ser adaptado pelo fabricante segundo a quantidade dos andares da residência ou do edifício onde o elevador será instalado.

\subsubsection{SENSORES DE PRESENÇA}

Bastante usado hoje em dia, para Furlan (2019), o sensor de presença é um dispositivo que pode ser utilizado de várias formas. Atualmente, esses dispositivos são utilizados, em sua maioria, em sistemas de iluminação e abertura de portas. Dessa forma, a princípio, esse sensor capta a presença de pessoas e por meio de um sinal o mesmo ativa a iluminação do local ou então abre a porta do recinto, permitindo a entrada e a saída das pessoas. Tal dispositivo já é utilizado por Aeroportos e Shoppings Centers, sobretudo em portas.

\subsubsection{MONITORAMENTO VIA INTERNET}

De acordo com Nogueira (2018), esse sistema possibilita o monitoramento de uma determinada residência de qualquer lugar do planeta. Basta ter acesso a internet. Especialmente em residências onde moram somente idosos, através desse sistema, todos os cômodos da residência podem ser monitorados 24 horas por dia. $\mathrm{O}$ que promove uma segurança maior, pois na eventualidade da ocorrência de algum acidente com o idoso que o impossibilite de pedir ajuda a alguém, o usuário desse sistema pode visualizar o que aconteceu para tomar as providências necessárias para socorrer a vítima. Esse sistema também pode ser utilizado por um celular que tenha acesso a internet.

\subsubsection{SISTEMA AUTOMATIZADO DE IRRIGAÇÃO}

Tendo um sistema automatizado de irrigação, o idoso ou a pessoa com deficiência não terá problemas em irrigar e cuidar do seu jardim. Acidentes como escorregões, devido ao chão molhado serão prevenidos e, ainda, o cansaço será evitado, podendo o morador usar esse tempo e energia em outras atividades. (RANGEL, 2013).

$\mathrm{RC}: 81825$

Disponível em: https://www.nucleodoconhecimento.com.br/educacao/domotica 


\subsubsection{PAINEL PARA CAMA}

Conforme Nichele (2013), o painel para cama é um sistema bastante útil, principalmente aos enfermos. Estes geralmente ficam nas cabeceiras das camas e possuem controles manuais. Ainda que bastante usados em motéis, estes painéis possuem grande utilidade aos pacientes que por alguma causa não podem se levantar da cama. Assim sendo, os botões são configurados a partir das necessidades de cada usuário, possibilitando a comodidade e o conforto, dois grandes benefícios desse sistema.

Hoje em dia não é muito difícil e se ver pessoas que após anos e anos de serviço ao seu país, ao seu governo, anos e anos cuidando de seus filhos, trabalhando duro para sobreviver e criar seus filhos, são largados em asilos para ali passarem o resto de seus dias, muitas vezes sozinhos e com pouco amparo, a tecnologia surge para amenizar isso, para trazer conforto, alegria, praticidade, uma vida melhor para quem já fez tanto por tantos. Assim também para pessoas com deficiências e que pessoas que necessitam de cuidados mais especializados.

\section{CONCLUSÃO}

O desenvolvimento do presente estudo possibilitou-nos atestar que a domótica é um tema que vem tendo cada vez mais ênfase e entrando em grandes pautas, muitas inovações e novas tecnologias dentro da área foram criadas recentemente e essa é uma tendência que só tem a se manter e aumentar. Além de trazer mais conforto, praticidade e facilidade para as famílias e empresas, é um auxílio muitas vezes imprescindível para o cotidiano de idosos e pessoas com deficiência, a evolução e a importância que cada vez mais o mundo tem dado para a domótica.

A domótica voltada para idosos e deficientes tem proporcionada a possibilidade de pessoas que antes tinham dificuldades de fazer até coisas simples do cotidiano, agora não apenas podem fazer tais coisas, como também podem ir além disso, podem fazer coisas que antes não achavam que um dia conseguiriam. Além disso

$\mathrm{RC}: 81825$

Disponível em: https://www.nucleodoconhecimento.com.br/educacao/domotica 
verificou-se que é uma área promissora a se fazer investimentos e para se dedicar a estudá-la e trabalhar na mesma para trazer novos aperfeiçoamentos e inovações.

\section{REFERÊNCIAS}

ADAMI, Anna. Domótica, InfoEscola, 7 de março de 2011. Disponível em: https://www.infoescola.com/tecnologia/domotica/. Acesso em: 11/04/2019.

AURESIDE, Associação Brasileira de Automação Residencial. Relatório Especial: O Mercado de Automação Residencial, 2012.

BELEZA, José Eduardo do Monte Moreira da Rocha. Sistema integrado de segurança e domótica, Faculdade de Engenharia da Universidade do Porto, janeiro de 2009. Disponível em: https://repositorioaberto.up.pt/bitstream/10216/59558/1/000135103.pdf.

BERNINI, Raphael de Almeida. SANTOS, Eduardo Ferro dos. Habitações inteligentes para pessoas com deficiência e de melhor idade baseada no programa minha casa minha vida, 1 Congresso Internacional de Ergonomia Aplicada Blucher Engineering Proceedings São Paulo, v.3, n.3, 13 dezembro de 2016.

Disponível em:

http://pdf.blucher.com.br.s3-sa-east1.amazonaws.com/engineeringproceedings/conaerg2016/7409.pdf.

DINO - Divulgador de notícias. Automação residencial possibilita mais autonomia para idosos e portadores de necessidades especiais, 3 de setembro de 2014. Disponível em: http://www.dino.com.br/releases/automacaoresidencialpossibilita-mais-autonomia-para-idosos-e-portadores-denecessidadesespeciais-dino89040740131.

DOMINGUES, Ricardo Gil. A domótica como tendência na habitação: Aplicação em habitações de interesse social com suporte aos idosos e incapacitados. Dissertação (Mestrado em Engenharia Urbana), Programa de Engenharia Urbana,

RC: 81825

Disponível em: https://www.nucleodoconhecimento.com.br/educacao/domotica 
Escola Politécnica, Universidade Federal do Rio de Janeiro, Rio de Janeiro, 2013. Disponível em: http://www.dissertacoes.poli.ufrj.br/dissertacoes/dissertpoli988.pdf

ELOY, Sara. PLÁCIDO, Isabel. DUARTE, José Pinto. NUNES, Renato. Utilização de domótica na estratégia de sustentabilidade social e ambiental, Proceedings of the 1ํ Congresso Internacional de Habitação no Espaço Lusófono (CD) ISCTE IUL, 8 de novembro de 2015. Disponível em: http://repositorio.Inec.pt:8080/jspui/bitstream/123456789/1001418/1/CIHEL_SE\%2bl P\%2bRN\%2bJPD.pdf.

FERREIRA, Victor Zago Gomes. A domótica como instrumento para a melhoria da qualidade de vida dos portadores de deficiência. Instituto Federal De Educação, Ciência E Tecnologia Da Paraíba. Diretoria De Ensino Departamento De Ensino Superior Curso Superior De Tecnologia Em Automação Industrial, João Pessoa, PB, 2010. Disponível em: https://d1wqtxts1xzle7.cloudfront.net/42836478/A_DOMOTICA_COMO_INSTRUME NTO_PARA_A_MELHORIA_DA_QUALIDADE_DE_VIDA_DOS_PORTADORES_DE _DEFICIENCIA.pdf?1455891065=\&response-contentdisposition=inline\%3B+filename\%3DA_DOMOTICA_COMO_INSTRUMENTO_PARA A_MELHO.pdf\&Expires=1615764983\&Signature=Kktq2LoxL5PypcLvD3 X3vczKpq QAPUN RmoxDgqCH1-q0TrtKsD5Uii3k-

TmZTnoY35FG6OEP6CvmHKOjP103O3pSTJk8mfe6xYPIwwELbCB3EaWCwCOAz MLthgKqenBeOJZxYVYp2cEYtLNa693ZBa0wnh9gde9egIXb5oUZ7F17dDjf2ECpbffs 30qM7eUpZNit6BX8VJgK25c8-

G6Q9NSqAOfk vloQzH BjvNMmmNvAUDqHLS08IF-3 JCVhlm9zeucucv3FiOyRCkSvPwqldJh-HxELWXEqq5B0-

huu oU6jl56yC0zWcAKmix3vpkqCPMsMj9IEborK bA_\&Key-PairId=APKAJLOHF5GGSLRBV4ZA.

FURLAN, Luiza. Como ter uma casa inteligente, Empresa Júnior de Arquitetura e Engenharia da USP São Carlos, 15 de agosto de 2019. Disponível em: https://eescjr.com.br/blog/blog-casa-inteligente/

RC: 81825

Disponível em: https://www.nucleodoconhecimento.com.br/educacao/domotica 
JÚNIOR, José Ricardo Gomes dos Santos. COSTA, Lucia de Fátima Vieira da. TAVARES, Sayonara Alice Cirilo. SOUSA, Layse Castilho. PESSOA, Guilherme Afonso Pillon de Carvalho Alves. BEZERRA, Larissa Guedes. Projeto de domótica para melhor idade, Instituto Federal De Educação Ciência E Tecnologia Do Rio Grande Do Norte - Campus Parnamirim, 15 de fevereiro de 2015. Disponível em: http://sistemaolimpo.org/midias/uploads/3f36f2e7d6c050e93c05e572c3395e3a.pdf.

LINS, Vitor. MOURA, Waldson. Domótica: automação residencial, 15 de novembro de 2009. Disponível em: http://www.unibratec.edu.br/tecnologus/wpcontent/uploads/2010/12/lins_moura.pdf. Acesso em: 18/04/2019

MARIOTONI, C. A. e ANDRADE Jr., E. P., Descrição de Sistemas de Automação Predial Baseados em Protocolos PLC Utilizados em Edifícios de Pequeno Porte e Residências, Revista de Automação e Tecnologia de Informação. Volume 1, número 1, 2007.

MARTINS, Rafaela. Conheça o poder da automação residencial para pessoa com deficiência, Freedom, 4 de dezembro de 2019. Disponível em: https://blog.freedom.ind.br/conheca-o-poder-da-automacao-residencial-para-pessoacom-deficiencia/.

NICHELE, Daniel Brandão. Automação residencial: um grande auxílio para idosos e deficientes, UNIVERSIDADE SÃO FRANCISCO Itatiba, 18 de maio de 2016. Disponível em: http://lyceumonline.usf.edu.br/salavirtual/documentos/1897.pdf

NOGUEIRA, Felipe Alberto da Silva. Monitoramento domiciliar de hábitos e ações de pessoas de terceira idade através de conceitos da Internet das Coisas, Universidade Federal Do Rio Grande Do Sul Instituto De Informática Curso De Engenharia De Computação, Porto Alegre, 2018. Disponível em: https://www.lume.ufrgs.br/bitstream/handle/10183/190154/001088828.pdf?sequence $=1 \&$ isAllowed $=\mathrm{y}$.

RC: 81825

Disponível em: https://www.nucleodoconhecimento.com.br/educacao/domotica 
NUNES, Renato Jorge Caleira. Análise comparativa de tecnologias para domótica, INESC-ID, 2002. Disponível em: http://www.domobus.net/docs/02JEACI02.pdf.

PAIVA, Evangelina de. NORMANDIA, Priscila. CÁSSIA, Rita de. ALVES, Rogério. JESUS, Rogério de. DIRANI, Ely. Domótica para idosos, Pontifícia Universidade Católica de São Paulo - PUC-SP, 18 de outubro de 2008. Disponível em: http://bt.fatecsp.br/system/articles/813/original/120-evangelina.pdf.

PERRACINI, Monica Rodrigues. Prevenção e manejo de quedas no idoso, 2002. Disponível

em: https://www.nescon.medicina.ufmg.br/biblioteca/imagem/artigo_prevencao_e_manej o_de_quedas_no_idoso_-_monica_rodrigues_perracini.pdf

RANGEL, Leonardo Siqueira. Aplicabilidades da automação residencial para população idosa: um estudo de caso, Universidade Candido Mendes - UCAM Programa De Pós-Graduação Em Engenharia De Produção Curso De Mestrado Em Engenharia De Produção, Campos Dos Goytacazes, RJ Setembro de 2013. Disponível em: https://pep.ucam-campos.br/wpcontent/uploads/2014/11/Leonardo_Siqueira.pdf.

SANTOS, Daniel Delano dos. SANTOS, José Emerson Oliveira. SILVA, Tassiana Lisboa Vieira da. OLIVEIRA, Thalya Lima de. DORIA, Nara Strappa Facchinetti, Automação residencial utilizando Arduino, Instituto Federal De Ciências e Tecnologia de Sergipe, outubro de 2016. Disponível em: http://sistemaolimpo.org/midias/uploads/3fb615cfbbbd00a7f693d3050c96893e.pdf.

SGARBI, Júlio André. TONIDANDEL, Flavio. Domótica inteligente: automação residencial baseada em comportamento, Centro Universitário da Fei São Bernardo do Campo, 6 de agosto de 2005. Disponível em: https://fei.edu.br/ flaviot/pub_arquivos/WTDIA06.pdf. Acesso em: 18/04/2019.

RC: 81825

Disponível em: https://www.nucleodoconhecimento.com.br/educacao/domotica 
TEIXEIRA, Álvaro José Carvalho. Casas inteligentes: adaptação desta tecnologia para deficientes e idosos, Universidade do Porto Faculdade de Engenharia Departamento de Engenharia Eletrotécnica e de Computadores, Tese de Mestrado em Engenharia Eletrotécnica e de Computadores, 4 de agosto de 2015.

Enviado: Novembro, 2020.

Aprovado: Março, 2021. 